\title{
Enhancements to the Short Pulse High Intensity Nanosecond X-radiator (SPHINX) Pulsed Power System
}

\author{
Nathan R. Joseph ${ }^{\xi}$, Mark E. Savage, Jacob C. Stephens, John A. Lott, Barbara A. Lewis, Rayburn D. \\ Thomas, Matthew A. Torres, Edward G. Holman \\ Sandia National Laboratories, Albuquerque, ${ }^{*}$ NM 87185 USA
}

\begin{abstract}
The Sandia National Laboratories SPHINX accelerator is used to study the response of electronics to pulsed $x$ ray and electron environments. The system consists of a Marx generator and an oil-insulated pulse-forming line with self-closing oil switches. SPHINX has a peak load voltage of $2 \mathrm{MV}$ and an adjustable pulse width ranging from 3 to $10 \mathrm{~ns}$. The previous pulsed-power system had reliability and triggering issues with the Marx generator and subsequent undesired variations in voltage output. SPHINX was upgraded to a new Marx-generator system that has solved many of the voltage-output fluctuation and timing issues. The new Marx generator uses recently developed lowinductance $100-\mathrm{kV}$ capacitors and $200-\mathrm{kV}$ spark-gap switches. This paper provides an overview of SPHINX while capturing in detail the design, characterization, and comparative performance of the new Marx generator.
\end{abstract}

\section{INTRODUCTION}

The Short Pulse High Intensity Nanosecond X-radiator (SPHINX) accelerator is used to study the response of electronics to pulsed X-ray and electron environments. SPHINX was designed and built by Pulse Sciences, Inc. and delivered for use to Sandia National Laboratories in January of 1986. The compact pulsed-power design allows SPHINX to have an adjustable output pulse width from 3 to $10 \mathrm{~ns}$ at a 2-MV peak. The original intention of SPHINX was to be a pulsed highpower microwave system. SPHINX is now used as an electron beam and bremsstrahlung accelerator with the capability of producing 12 shots a day.

In January of 2014, the SPHINX accelerator began to show indications of issues with the pulsed-power system. An indepth analysis, conducted using shot data, determined that the Marx generator consistently produced undesired variation in both power output and timing. The goal was to achieve a substantial decrease in the $+/-1.83-\mathrm{GW}(18.3 \%$ of the nominal $100 \mathrm{GW}$ peak power) and +/-210-ns output variations. Output variations in peak power cause fluctuations in radiation output which have negative effects on understanding experimental data. The timing variations can cause experimental data to be lost. The improvements would be targeted to output variations in peak power and timing of the SPHINX system. The end result was a complete redesign of the SPHINX Marx generator.

\footnotetext{
* Sandia National Laboratories is a multi-program laboratory managed and operated by Sandia Corporation, a wholly owned subsidiary of Lockheed Martin Corporation, for the U.S. Department of Energys National Nuclear Security Administration under contract DE-AC04-94AL85000.

$\xi$ email: nrjosep@sandia.gov
}

\section{SySTEM MODIFICATION AND DESIGN IMPROVEMENT}

The new Marx system capitalizes on recent technological advancements in manufacturing and design by utilizing lowinductance, $100-\mathrm{kV}$ capacitors and 200-kV spark-gap switches [1]. Like its predecessor, the new Marx generator uses sulfur hexafluoride $\left(\mathrm{SF}_{6}\right)$ as an insulating gas in the spark-gap switches. The back-to-back design of the new switch and capacitor configuration (Fig. 1) reduces the inductance of the SPHINX Marx generator from $7.1 \mu \mathrm{H}$ to $3.6 \mu \mathrm{H}$.

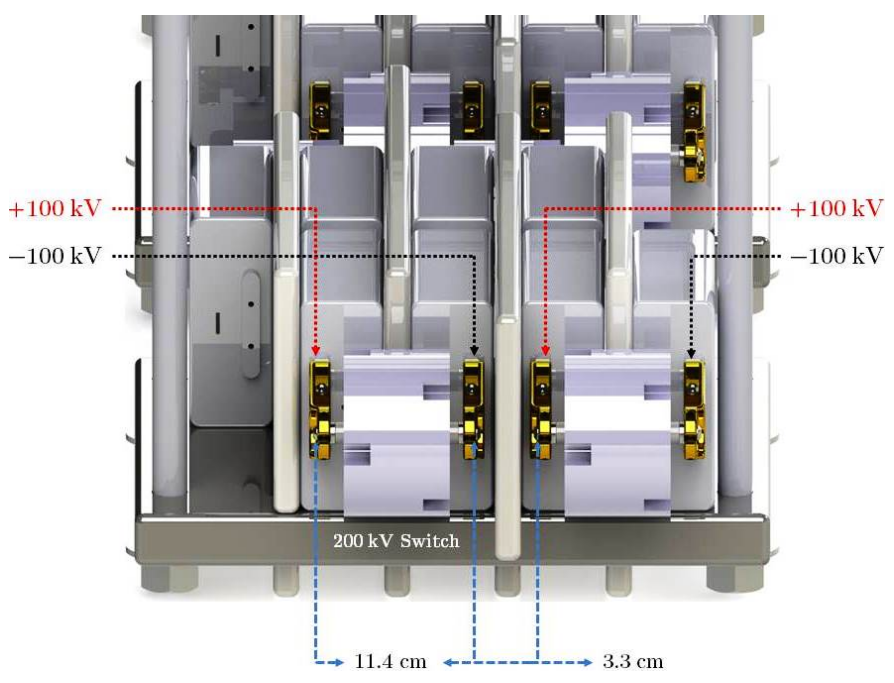

Fig. 1: SPHINX Marx Configuration

The close proximity of components in the Marx generator is achievable by the use of corona shields on the spark gaps and nylon isolation plates between capacitors. The corona shields reduce field-enhancement points. As a safety measure, an energy diverter with a pre-determined oil break-down gap was affixed to the output arm of the Marx generator, dissipating late-time reflections. The isolating effects of the corona shields, coupled with the addition of the energy diverter cause less damage to the SPHINX Marx components overall.

To decrease timing jitter, the SPHINX Marx triggering circuit was also redesigned. The new circuit triggers the first four spark-gap switches. Each spark-gap mid-plane electrode is individually attached to a $15-\mu \mathrm{H}$ inductor. An inductor allows for ringing gain to occur on the incoming triggering pulse. 
This increases the field enhancement on the switch mid-plane electrodes, resulting in less switch closure timing jitter.

The change in the configuration of the new Marx required replacement of the previous high-voltage charging system. The previous Marx generator had 18 stages of capacitors at a value of $64 \mathrm{nF}$ per stage and was charged at $+/-50 \mathrm{kV}$. The redesigned Marx generator has 24 stages of capacitors at a value of $40.6 \mathrm{nF}$ per stage and is charged a $+/-100 \mathrm{kV}$. The redesigned Marx generator required a charging system of $+/-$ $100 \mathrm{kV}$ to equal the previous Marx generator output. The reengineered charging system replaced an antiquated pneumatic system with Ross relays (Fig. 2). A capacitive filtering circuit was also added to dampen pulse reflections that can occur when the system is fired.

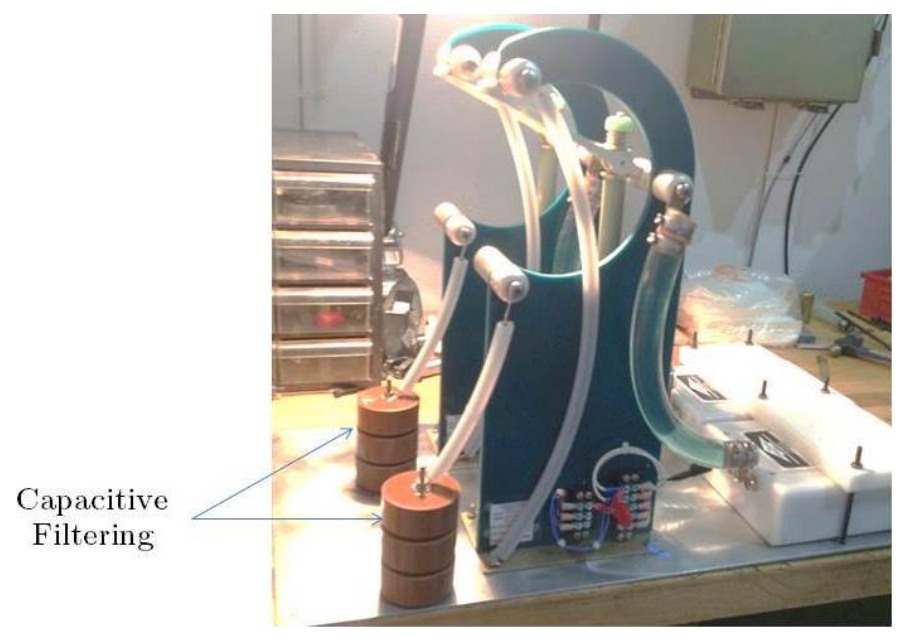

Fig. 2: SPHINX Ross Relay System

\section{CHARACTERIZATION}

Before the new Marx installation, SPHINX had little characterization data to be found for the five diagnostics that existed on the machine (listed following this paragraph). With the implementation of the new Marx generator an additional Rogowski coil diagnostic[5] was added, and a recharacterization of the accelerator was conducted. The following lists components of the testing procedure:

- Identify what diagnostics will be used for full system characterization shots.

- Marx current-viewing resistor[4] (CVR).

- Rogowski coil for diagnosing Marx total output current.

- Capacitive-voltage monitor(s)[2](V-dot) on pulse forming line (PFL)/peaking switch.

- Current shunt monitor[3] on PFL/peaking switch.

- Capacitive-voltage monitor on magnetically insulated transmission line (MITL).

- Current shunt monitor on MITL.

- Develop method for characterizing capacitive voltage monitors on PFL.

- Develop method for characterizing Rogowski coil.
- Conduct self-break tests on Marx generator to determine the $\mathrm{SF}_{6}$ break down pressure curve.

- Conduct Marx-generator short-circuit shots.

- Conduct SPHINX full-system short-circuit shots at various charge voltages.

- Conduct SPHINX ring-over shot at lowest charge voltage $/ \mathrm{SF}_{6}$ pressure achievable.

\section{A. Capacitive Voltage Monitor Characterization}

SPHINX has three capacitive voltage monitors located on different sections of the PFL (Fig. 3). VP2 (Voltage Pulse Line 2) is located at the beginning of the $25 \Omega$ PFL. VP2 was switched from a self integrating monitor to a differential monitor at the beginning of the characterization tests. VO2 (Voltage Gap Opening 2) is located at the $25 \Omega$ to $40 \Omega$ PFL adjustable gap and is a self-integrating monitor. VO3 (Voltage Gap Opening 3) is located at the peaking switch and is a selfintegrating monitor.

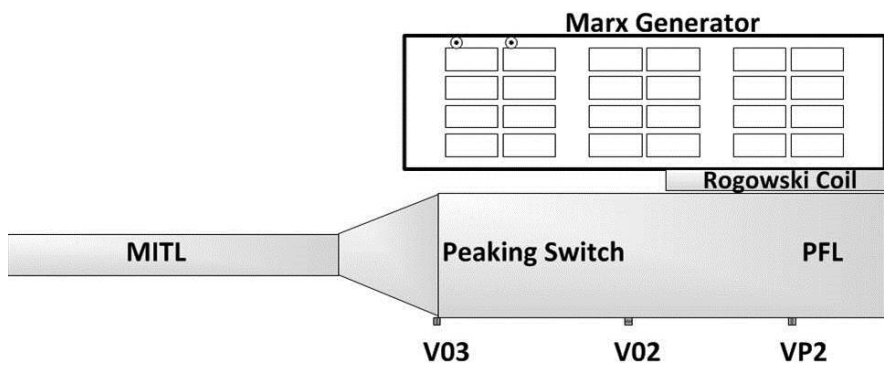

Fig. 3: SPHINX Diagnostics Configuration

Each monitor was characterized using the following method:

- $\quad$ Connect $4 \mathrm{kV} 2 \Omega 40 \mathrm{~ns}$ pulser to input PFL.

- Insert $14.89 \mathrm{k} \Omega$ resin-filled resistor into PFL rod holder until contact is made.

- $\quad$ Ensure PFL rod holder is on same circumference as capacitive voltage monitor.

- Using a resistance meter, measure between resistor output and input PFL. Meter should read $14.89 \mathrm{k} \Omega$. If meter does not read $14.89 \mathrm{k} \Omega$ readjust resistor and measure again.

- Clamp ground braid onto outside of resistor and onto a non-painted surface on SPHINX. This will ensure good signal coupling and reduce distortion on recorded signal.

- Connect Heliax cable to N-connector output of resistor, and connect other Heliax cable to output of capacitive voltage monitor.

- Use a factor of $100-\times$ attenuation at both scope channels. Be sure that each attenuator and cable has been properly characterized using a network analyzer.

- Connect Heliax cables to attenuators and connect attenuators to digital oscilloscope. 
- In the acquisition system software add correction factors for any differences between attenuators and cable compensations.

- Trigger system and record data.

- Integrate capacitive voltage monitor signal (if monitor is not self-integrating).

- Conduct least squares comparison between resistive monitor and capacitive voltage monitor.

- The least-squares output will give a fit ratio between the resistive and capacitive monitor signal. The ratio given by the least squares is the multiplication correction or (gauge factor) for the capacitive voltage monitor signal (Fig. 4).

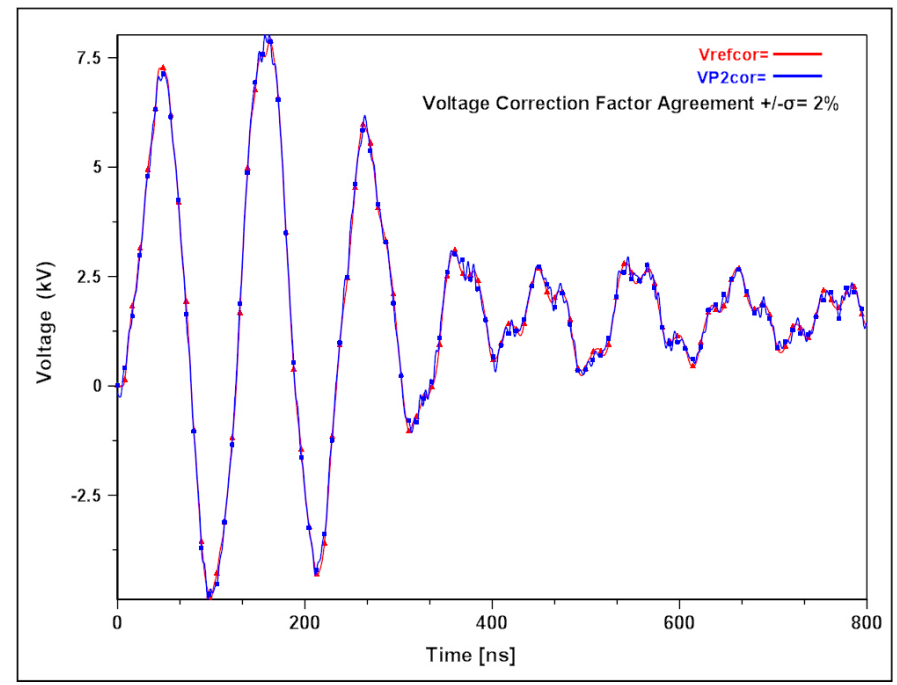

Fig. 4: SPHINX Capacitive Voltage Monitor Characterization

Monitor signals were compared to reference monitors built as resistive dividers. The resistor values were measured with a Fluke multimeter and were within $1 \%$ of the nameplate value. The Fluke meter is then the reference standard for the system. The characterizations included a monitor for the V-dot on the first pulse-charged section of coax line and a self-integrating voltage monitor located just upstream of the first self-breaking oil switch. Some of the original SPHINX monitors were selfintegrating.

This is not a great advantage with todays fast digitizers, and the act of self-integrating reduces the signal substantially. This is essentially attenuating and integrating the signal outside the screen box, and is not generally good practice because of electrical noise. While self-integrating monitors can be important for very fast signals (rise time comparable to the digitizer sampling time), this is less important with the fast digitizing rates common today. The drawback of self-integrating monitors is the inescapable droop when passive components are used. The droop can be readily corrected with a function of Equation (1).

$$
V_{\text {correction }}=V_{\text {raw }}+\frac{1}{\tau} \int_{-\infty}^{t} \mathrm{~V}_{\text {raw }}\left(t^{\prime}\right) \mathrm{d} t^{\prime}
$$

\section{B. Rogowski Coil Monitor Characterization}

SPHINX recently added a Rogowski-coil diagnostic that is fitted around the junction between the Marx generator and the PFL. The Rogowski coil is secured by a nylon plate with the coil facing the ground plane of the SPHINX oil tank. The method used to characterize the Rogowski coil is as follows:

- $\quad$ Remove pulse forming line pneumatic shorting clamp.

- $\quad$ Run all sixteen lines in a loop around a Pearson coil[6] with at least $25 \mathrm{~ns}$ rise time response.

- $\quad$ Connect $4 \mathrm{kV} 2 \Omega 40 \mathrm{~ns}$ pulser to input PFL.

- Connect Heliax cable to N-connector output of Pearson coil and connect other Heliax cable to output of Rogowski coil.

- Use a factor of $100-\times$ attenuation at both scope channels. Be sure that each attenuator and cable has been properly characterized using a network analyzer.

- Connect Heliax cables to attenuators and connect attenuators to digital oscilloscope.

- In the acquisition system software add correction factors for any differences between attenuators and cable compensations.

- Trigger system and record data.

- Integrate Rogowski-coil data.

- Conduct least-squares comparison between Pearson coil and Rogowski-coil data.

- The least-squares output will give a ratio between the Pearson and Rogowski coil. The ratio given by the least squares is the multiplication correction factor for the Rogowski coil.

The signal from the Rogowski coil was compared to a reference Pearson coil with a known response and voltage-tocurrent ratio. Thus the Pearson coil was used as the reference standard for the system.

\section{Self-Break Tests}

After the new Marx-generator installation, a series of self-break tests were conducted. The self-break test provides information on the correct gas pressure setting for a given charge voltage. The resulting data showed what $\mathrm{SF}_{6}$ pressures are needed for a chosen charge voltage. The $\mathrm{SF}_{6}$ pressure in the Marx switches were set at 8, 10, 12 and 14 psi. The high-voltage power supplies were set to $+/-95 \mathrm{kV}$. Four selfbreak tests at each pressure setting were conducted. The powersupply voltage-monitor readings were then documented at the time of self-break. The self-break curve results are shown in (Fig. 5). The $80 \%$ and $60 \%$ self-break curves are interpolated values from the $100 \%$ self-break curve. 


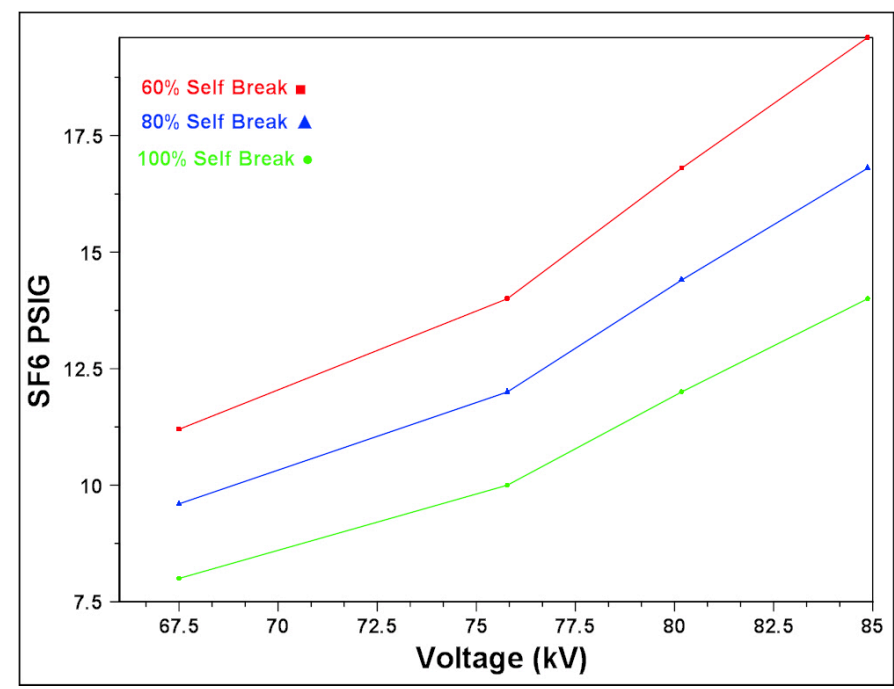

Fig. 5: SPHINX Self-Break Curve

\section{Short-Circuit Tests}

To diagnose the output pulse shape and current of the new Marx generator, a series of short-circuit shots were conducted on the SPHINX pulsed-power system. The Marx charge voltages were set to $+/-50,70$ and $80 \mathrm{kV}$. The PFL and peaking switch were shorted through the output transmission line to complete a short-circuit configuration. A simplified pulsedpower circuit model of SPHINX was used to produce the RLC Approx waveform in (Fig. 6). The RLC Approx waveform overlays the Marx $C V R$ experimental data which coorelates to each other within $+/-3 \%$.

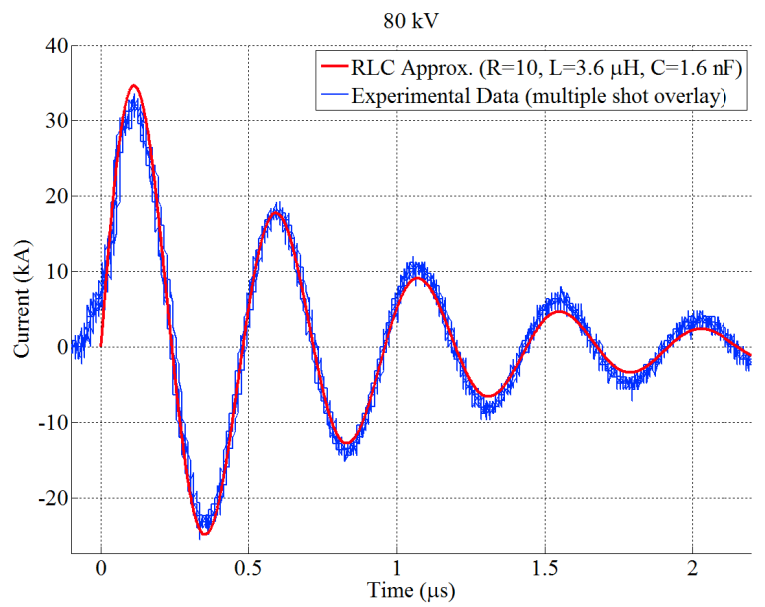

Fig. 6: SPHINX +/-80 kV Short-Circuit Shot

\section{E. Ring-Over Tests}

A ring-over test of the SPHINX PFL was conducted at a Marx charge voltage of $+/-32 \mathrm{kV}$ and a $\mathrm{SF}_{6}$ pressure of 5 psi. From the test data, the SPHINX PFL capacitance can be measured. Using the Rogowski-coil output current (Fig. 7a) and integrating (Fig. 7a) using Equation (2)[6] the total charge of the SPHINX PFL can be found (Fig. 7b). With finding peak charge of Rogo(Q) (Fig. 7b) and taking the peak voltage from the VP2 PFL voltage monitor (Fig. 8), the total capacitance of the SPHINX PFL can found using Equation (3)[4] and Equation (4)[4].

$$
\begin{aligned}
& \operatorname{Rogo}(Q)=\int_{0}^{t} \operatorname{Rogo}(I) \\
& Q_{\mathrm{PFL}}=C_{\mathrm{PFL}} * V P 2_{\text {Peak }} \\
& C_{\mathrm{PFL}}=Q_{\mathrm{PFL}} / V P 2_{\text {Peak }}
\end{aligned}
$$

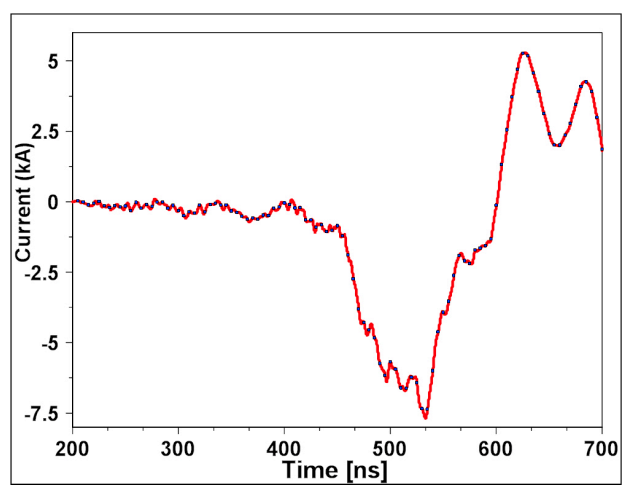

(a) Rogowski Coil Current Waveform

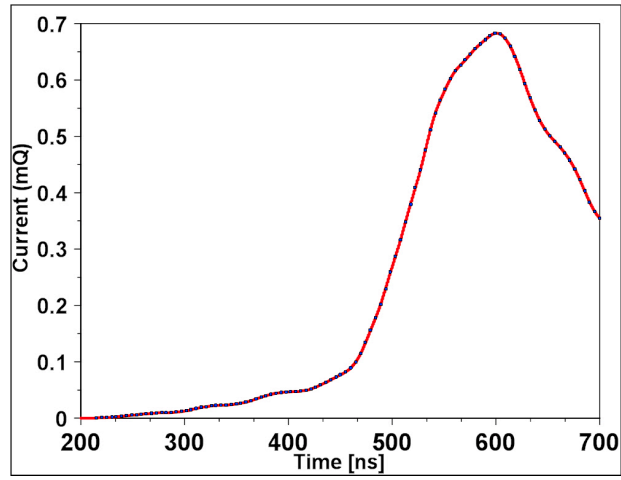

(b) Rogowski Coil Charge Waveform

Fig. 7: SPHINX Marx Triggering System

When the measured values of $\mathrm{Q}_{\mathrm{PFL}}$ and $\mathrm{VP} 2_{\text {Peak }}$ are placed into Equation (4)[4], a total of $9.34 \mathrm{nF}$ is found for the SPHINX PFL capacitance. 


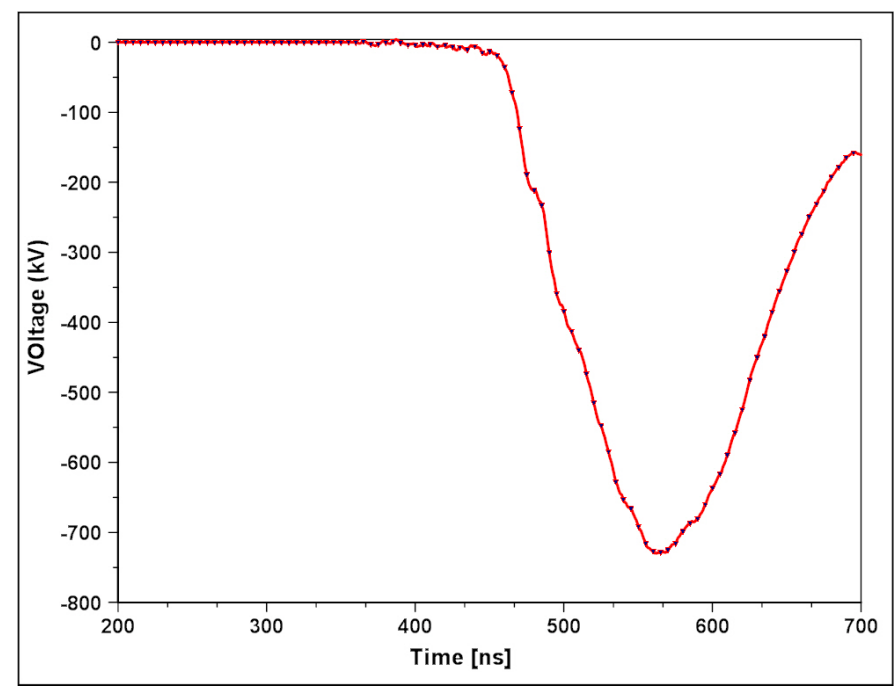

Fig. 8: VP2 Voltage Monitor Ring-Over Waveform

\section{COMPARISON}

A five-shot series of a known SPHINX configuration was conducted. The series would show the enhancements of the redesigned SPHINX Marx-generator versus the previous. Table I shows the comparison of the previous and redesigned Marx generator total erected capacitance, inductance, and charge voltage. Table II shows the comparison of power output and timing jitter. The comparisons are in terms of power output and timing jitter to one sigma.

TABLE I: SPHINX Marx Values for Comparison Testing

$*$ Relative charge voltage to $+/-100 \mathrm{kV}$

\begin{tabular}{|l|l|l|l|}
\hline SPHINX Marx Configuration & Erected Capacitance & Inductance & Charge Voltage \\
\hline Previous Marx & $3.6 \mathrm{nF}$ & $7 \mu \mathrm{H}$ & $+/-50 \mathrm{kV}^{*}$ \\
\hline Redesigned Marx & $1.6 \mathrm{nF}$ & $3.6 \mu \mathrm{H}$ & $+/-80 \mathrm{kV}$ \\
\hline
\end{tabular}

TABLE II: SPHINX Total Output Comparison

\begin{tabular}{|l|l|l|}
\hline SPHINX Marx Configuration & Power Output Jitter $+/-\sigma$ & Timing Jitter $+/-\sigma$ \\
\hline Previous Marx & $18.3 \%$ & $210 \mathrm{~ns}$ \\
\hline Redesigned Marx & $0.22 \%$ & $20.2 \mathrm{~ns}$ \\
\hline
\end{tabular}

\section{SUMMARY}

The results from section IV were taken using the same load voltage and current monitors for both redesigned and previous SPHINX Marx shot data. The total power output deviations in Table II are based upon an nominal power output of $100 \mathrm{GW}$. The timing output jitter in Table II was calculated by taking a standard deviation from the peak load voltage times. The comparison data shows a reduction from $18.3 \%$ to $0.22 \%$ in power output jitter and a reduction of $+/-189 \mathrm{~ns}$ in timing jitter. The concentrated efforts in the characterization of SPHINX, has led to an understanding of and enhanced performance for the SPHINX accelerator.

\section{REFERENCES}

[1] J. R. Woodworth, J. A. Alexander, F. R. Gruner, W. A. Stygar, M. J Harden, J. R. Blickem, G. J. Dension, F. E. W. L. M. Lucero, H. D. Anderson, L. F. Bennett, S. F. Glover, D. Van DeValde, and M. G. Mazarakis, "Low-inductance gas switches for linear transformers," Phys. Rev. ST Accel. Beams, vol. 12, no. 6, Jun. 2009.

[2] P. Choi and M. Favre, "A fast capacitive voltage monitor for low impedance pulse lines," Digest of Technical Papers, vol. 2, pp. 880-885, Sep. 1995

[3] J. L. Pack and I. Liberman, "Voltage and current measurements for fast pulsed high current discharges," Review of Scientific Instruments, vol. 52, pp. 1580-1582, Oct. 1981.

[4] S. Pai and Q. Zhang, Introduction to High Pulse Technology, 1995.

[5] D. G. Pellinen, M. S. di Capua, S. E. Sampayan, H. Gerbracht, and M. Wang, "Rogowski coil for measuring fast, high-level pulsed currents," Review of Scientific Instruments, vol. 51, pp. 1535-1540, Nov. 1980.

[6] H. Bluhm, Pulsed Power Systems: Principles and Applications, 2006.

\section{APPENDIX}

The 1986 SPHINX pulsed-power accelerator design consisted of a simplistic and versatile architecture that leveraged many fundamental, cutting-edge concepts of that time. The Marx generator was 18 stages of $64 \mathrm{nF}$ capacitors. The Marx generator erected capacitance was $3.6 \mathrm{nF}$. The output of the Marx was coupled into an adjustable-gap PFL that connected to an adjustable-gap peaking switch. The peaking switch connected to a crowbar gap, linked to an oil/vacuum insulator. The oil/vacuum insulator was coupled to a MITL. The Marx generator was a dual-polarity design that had a maximum bias voltage of $+/-50 \mathrm{kV}$ per stage. The PFL was comprised of two hemispherical electrode geometries, set at 25 and $40 \Omega$. The two segments were bridged by an oil-insulated switch gap, engineered to allow for a 0 to $6.3-\mathrm{cm}$ gap adjustment. The peaking switch consisted of 30 (two sets of 15) stainless-steel radius-tipped rods and provide a 0 to $3.81-\mathrm{cm}$ gap adjustment. The upstream power would then flow into the peaking-switch rods and pass through the pre-pulse plate. The pre-pulse plate consisted of $154.1-\mathrm{cm}$ holes intended to hold off the initial wave-pulse foot. A crowbar, situated on the output side of the peaking switch, was intended to act as either a late-time reflection diverter or as part of the pulse-shaping circuit. The crowbar afforded additional switch-gap modifications from 0 to $2.54 \mathrm{~cm}$. The output line of the crowbar was connected to a $60 \Omega$ tapered vacuum insulator. The outer-to-inner section of the vacuum insulator tapered from a $60.96-\mathrm{cm}$ diameter to a $30.48-\mathrm{cm}$ diameter at the coupling point of the coaxial vacuum line. The line was extended by two aluminum tubes that acted as a MITL. The inner MITL was $304.8 \mathrm{~cm}$ in length and 11.43 $\mathrm{cm}$ in diameter. The outer MITL was $304.8 \mathrm{~cm}$ in length and $30.48 \mathrm{~cm}$ in diameter. Both tubes run parallel to each other thus preserving the coaxial transmission-line geometry. The calculated impedance of the MITL is $58.8 \Omega$, closely matching the impedance of the vacuum insulator. 